\title{
Prospective Clinical Trial of Intravitreal Aflibercept Treat-and- extend Regimen for Diabetic Macular Edema: 1-Year Outcomes
}

\author{
Hiroki Mieno ${ }^{1}$, Kazuhito Yoneda ${ }^{1}$, Nobuhiro Terao ${ }^{1}$, Kengo Yoshii $^{2}$, Kentaro Kojima $^{1}$, Kenji Nagata $^{1}$, Chie Sotozono $^{1}$ \\ ${ }^{1}$ Department of Ophthalmology, Kyoto Prefectural University of Medicine, Kyoto, Japan \\ ${ }^{2}$ Department of Mathematics and Statistics in Medical Sciences, Kyoto Prefectural University of Medicine, Kyoto, Japan
}

\begin{abstract}
Purpose: To investigate the efficacy of aflibercept for the treatment of diabetic macular edema via a treat-and-extend regimen.

Methods: This prospective, single-center, open-label, interventional study involved 30 patients with a best-corrected visual acuity (BCVA) $\leq 0.6$ and a central retinal thickness $(C R T) \geq 300 \mu \mathrm{m}$. The enrolled eyes each received a monthly intravitreal aflibercept injection until the CRT decreased below $300 \mu \mathrm{m}$, upon which the administration interval was extended for 1 month until the CRT once again increased to $\geq 300 \mu \mathrm{m}$. Main outcome measures were median BCVA and CRT at 6 and 12 months after initiation of treatment via last observation carried forward analysis, the median number of injections over the 12 months, and the effects on the diabetic retinopathy severity scale (DRSS) score of the patients who completed the 12-month follow-up period.

Results: Of the 30 enrolled patients, 29 and 25 respectively completed the 6- and 12-month follow-up examinations. From baseline to 6 and 12 months after treatment initiation, the median BCVA (logarithm of the minimum angle of resolution) significantly improved from 0.52 to 0.30 and 0.35 , respectively, and the median CRT significantly decreased from 439.5 to 268.5 and $249.0 \mu \mathrm{m}$, respectively. The median number of injections over the 12-month follow-up period was 6.0. Compared to baseline, the DRSS score at 12 months was improved by 2 steps in $16 \%$ of patients; in no cases did the DRSS score worsen or improve by three steps or more.
\end{abstract}

Conclusions: When administered in a treat-and-extend regimen, aflibercept is an effective treatment option for diabetic macular edema.

Key Words: Aflibercept, Intravitreal injections, Macular edema

Received: January 3, 2020 Final revision: March 26, 2020

Accepted: April 10, 2020

Corresponding Author: Kazuhito Yoneda, MD, PhD. Department of Ophthalmology, Kyoto Prefectural University of Medicine, $465 \mathrm{Ka}$ jii-cho, Hirokoji-agaru, Kawaramachi-dori, Kamigyo-ku, Kyoto 6020841, Japan. Tel: 81-75-251-5578, Fax: 81-75-251-5938, E-mail: kazuyone@ koto.kpu-m.ac.jp

The part of this study was presented at EURETINA 2018 Congress, 2023 September, Vienna, Austria.
Diabetic macular edema (DME), a major diabetic retinopathy (DR)-related complication, severely reduces vision and can ultimately lead to blindness. Vascular endothelial growth factor (VEGF) is strongly associated with the onset of DME [1], and intravitreal anti-VEGF therapy is currently regarded as the gold standard treatment for DME [2-4].

Previous randomized clinical trials have illustrated the efficacy of anti-VEGF agents for the treatment of DME 
when administered monthly or bimonthly $[5,6]$, but such treatment regimens do not take into consideration the specific needs of individual patients, nor are they designed to match what is required in actual clinical settings. Prior to their application in the treatment of DME, anti-VEGF agents were approved for patients afflicted with neovascular age-related macular degeneration, for which various treatment regimens have been reported [7-9]. Among these, 'treat-and-extend' (TAE) regimens are often preferred because they are proactive, provide a more personalized approach, reduce treatment burdens, and greatly minimize the risk of vision loss $[3,10,11]$.

Aflibercept, an anti-VEGF agent that is a soluble decoy receptor drug, reportedly inhibits VEGF-A, VEGF-B, placental growth factor [12], and galectin-1 [13]. The Diabetic Retinopathy Clinical Research Network published a comprehensive comparative study of anti-VEGF agents for the treatment of DME, finding that aflibercept was the most effective anti-VEGF agent for improving vision, especially in patients having severe loss of visual acuity (VA) at baseline, namely those having VA of 20 / 50 or worse [2].

In the present study, we investigated the efficacy of aflibercept for the treatment of DME via a TAE regimen.

\section{Materials and Methods}

\section{Study design}

In this prospective, single-center, open-label, interventional study, 30 diabetes mellitus patients were enrolled at the Department of Ophthalmology, Kyoto Prefectural University of Medicine, Kyoto, Japan between March 2015 and
July 2017. The study protocols were approved by the institutional review board of Kyoto Prefectural University of Medicine (ERB-C-304-3); in accordance with the tenets set forth in the Declaration of Helsinki, written informed consent was obtained from all patients prior to enrollment. This study is registered with the UMIN Clinical Trials Registry (UMIN000022476).

\section{Patients}

The primary criteria for inclusion in this study were as follows: diabetes mellitus patient older than 20 years, DME involving the fovea, and DME-related loss of VA. Other key inclusion criteria were best-corrected visual acuity (BCVA) less than or equal to 0.6 and central retinal thickness (CRT) equal to or greater than $300 \mu \mathrm{m}$ as calculated by optical coherence tomography (OCT; 3D OCT-2000 or DCI OCT Triton, Topcon Corporation, Tokyo, Japan). If a patient's eyes both met the inclusion criteria, the data on the eye having the poorer pretreatment VA were used; if the pretreatment VA was the same in both eyes, the data on the eye having the greater pretreatment CRT were used.

Patients were excluded from the study if they met one of the following criteria: a history of vitreous surgery (including encircling buckling); any retinal coagulation treatment or treatment including injection of any anti-VEGF agent within 90 days prior to enrollment; active proliferative DR; a history of ocular inflammation; a history of intraocular surgery within 90 days prior to enrollment; aphakia; fluorescein allergy; glaucoma with poor control of intraocular pressure (defined as $>25 \mathrm{mmHg}$ ); myopia of -8 diopters or greater; current severe systemic infection; current treatment with carcinostatic agents; uncontrolled

Table 1. Study protocol

\begin{tabular}{|c|c|c|c|c|c|c|}
\hline & & & & & & \\
\hline & 0 (baseline) & 1 & $2-5$ & 6 & $7-11$ & 12 \\
\hline Visit & 0 & $\bigcirc$ & $\bigcirc^{*}$ & $\bigcirc^{\dagger}$ & $\bigcirc^{*}$ & $\bigcirc^{*}$ \\
\hline Aflibercept injection & $\bigcirc$ & $\bigcirc$ & $\bigcirc^{*}$ & $\bigcirc^{\dagger}$ & $\bigcirc^{*}$ & $\bigcirc^{*}$ \\
\hline BCVA & $\bigcirc$ & $\bigcirc$ & $\bigcirc^{*}$ & $\bigcirc^{+}$ & $\bigcirc^{*}$ & $\bigcirc^{*}$ \\
\hline OCT & $\bigcirc$ & $\bigcirc$ & $\bigcirc^{*}$ & $\bigcirc^{\dagger}$ & $\bigcirc^{*}$ & $\bigcirc^{\ddagger}$ \\
\hline Fundus photography & 0 & 0 & $\bigcirc^{*}$ & $\bigcirc^{\dagger}$ & $\bigcirc^{*}$ & $\bigcirc^{*}$ \\
\hline
\end{tabular}

$\mathrm{BCVA}=$ best-corrected visual acuity OCT $=$ optical coherence tomography.

*Depending on disease activity, treat and extend regimen; 'Primary endpoint, essential visit; ${ }^{\dagger}$ Secondary endpoint, essential visit. 
diabetes mellitus (defined as glycated hemoglobin $\geq 12 \%$ ); uncontrolled blood pressure (defined as systolic $\geq 180$ $\mathrm{mmHg}$ or diastolic $\geq 100 \mathrm{mmHg}$ while seated); a history of either cerebral vascular accident and/or myocardial infarction within 180 days prior to enrollment; renal failure requiring dialysis or renal transplantation; and current pregnancy or breast-feeding.

\section{Treatment regimen}

All enrolled eyes received $2 \mathrm{mg}$ intravitreal aflibercept injections (IAIs) according to the study protocol (Table 1). Each patient underwent an IAI at the initial visit and 1 month after the initial visit (i.e., month 1), at which time the TAE regimen was initiated. The criterion for extending the interval between aflibercept administrations was CRT decrease and maintenance below $300 \mu \mathrm{m}$. Namely, the enrolled eyes underwent IAI once per month until CRT decreased below $300 \mu \mathrm{m}$, after which the administration interval was extended for 1 month until the patient's CRT once again exceeded $300 \mu \mathrm{m}$, a condition termed recurrence. For patients showing recurrence, the administration interval was returned to the previous interval that did not lead to recurrence.

\section{Outcome measures}

In this study, the primary endpoint was median BCVA and CRT 6 months after initiation of treatment, and the secondary endpoint was median BCVA and CRT 12 months after initiation of treatment. BCVA was measured using a standard Japanese Landolt VA chart, and the decimal VA measured was converted to the logarithm of the minimum angle of resolution for statistical analyses. Additional outcomes included the number of IAIs administered until the CRT first decreased below $300 \mu \mathrm{m}$, the median number of IAIs administered during the 12 months, and the effects on DR. To evaluate the number of IAIs administered until CRT first decreased below $300 \mu \mathrm{m}$, patients for whom CRT never decreased below $300 \mu \mathrm{m}$ at the time of dropout were classified into a separate group. We investigated whether there was any observable difference in baseline characteristics among groups showing different numbers of IAIs administered until CRT first decreased below $300 \mu \mathrm{m}$. DR was evaluated using the Early Treatment Diabetic Retinopathy Study (ETDRS) diabetic reti- nopathy severity scale (DRSS) score [14] at baseline and at 6 and 12 months after initiation of treatment. DRSS scores were assigned by two retina specialists (HM and NT). Patients with a history of panretinal photocoagulation were graded as a minimum severity level of 60 ; such patients may worsen in retinopathy severity but, by definition, cannot improve to severity level less than 60 , as reported previously [15].

\section{Statistical analysis}

Last observation carried forward analysis was conducted for the primary and secondary endpoints. A complete case analysis was conducted to evaluate the median number of IAIs administered for 12 months and the progress of the DR. All statistical analyses were performed in R ver. 3.4.3 (R Foundation for Statistical Computing, Vienna, Austria). The primary and secondary endpoints were assessed using the Steel-Dwass test for multiple comparisons. To compare the baseline characteristics of subgroups requiring different numbers of IAIs until CRT first decreased below $300 \mu \mathrm{m}$, the Kruskal-Wallis test and the Fisher exact test were used as appropriate. A $p$-value less than 0.05 was considered statistically significant. Descriptive analysis of data was carried out using medians and interquartile ranges as appropriate.

\section{Results}

Of the 30 enrolled patients, $29(96.7 \%)$ completed the 6-month follow-up and 25 (83.3\%) completed the 12-month follow-up. Patients dropped out as follows: one patient at month 3 , two patients at month 7 , and two patients at month 8 . Table 2 summarizes the characteristics of enrolled patients at baseline. Regarding the ETDRS DRSS score, a DRSS score of 53 represents severe nonproliferative DR. As previously reported [16], the cutoff value of the DRSS score was set at 53 because the risk of developing proliferative DR is high in patients with a DRSS score of 53 or greater.

\section{VA and anatomic outcomes}

Median BCVA (logarithm of the minimum angle of resolution) significantly improved from 0.52 (interquartile 
Table 2. Demographics and baseline characteristics of the 30 patients

\begin{tabular}{lc}
\hline Characteristics & Value \\
\hline Age $(\mathrm{yr})$ & $69.5(63.0-74.0)$ \\
Sex, female & $10(33.3)$ \\
Phakia & $22(73.3)$ \\
HbA1c $(\%)$ & $6.85(6.50-7.65)$ \\
eGFR $\left(\mathrm{mL} / \mathrm{min} / 1.73 \mathrm{~m}^{2}\right)$ & $56.2(43.8-69.1)$ \\
History of PRP & $9(30.0)$ \\
DRSS score $\geq 53$ at baseline & $17(56.7)$ \\
\hline
\end{tabular}

Values are presented as median (interquartile range) or number (\%).

HbAlc $=$ glycated hemoglobin; eGFR = estimated glomerular filtration rate; $\mathrm{PRP}=$ panretinal photocoagulation; $\mathrm{DRSS}=$ diabetic retinopathy severity scale.

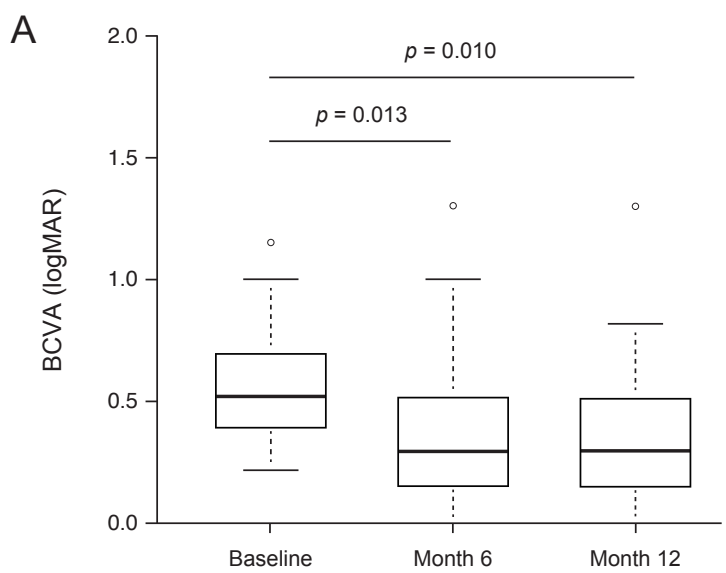

B

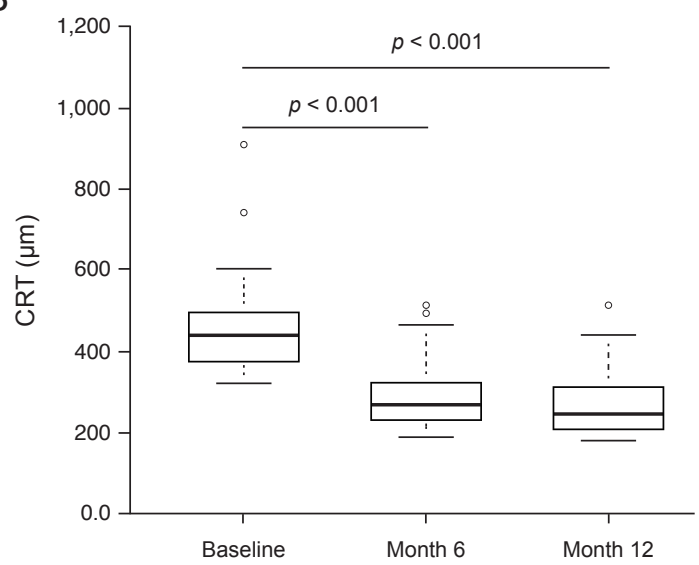

Fig. 1. The changes in (A) best-corrected visual acuity (BCVA) and $(B)$ central retinal thickness $(C R T)(n=30)$. The vertical-column boxes indicate values between the 25 th and 75 th percentiles (the central horizontal line within each box indicates the median). The circles denote outliers. The Steel-Dwass test for multiple comparisons was used to calculate the $p$-values. range, $0.40-0.70)$ at baseline to 0.30 (interquartile range, $0.15-0.52)$ at 6 months after initiation of treatment $(p<$ 0.05 ) and to 0.35 (interquartile range, $0.17-0.52$ ) at 12 months after initiation of treatment $(p<0.05)$. Median CRT significantly decreased from $439.5 \mu \mathrm{m}$ (interquartile range, $372.0-496.8 \mu \mathrm{m}$ ) at baseline to $268.5 \mu \mathrm{m}$ (interquartile range, $233.8-325.5 \mu \mathrm{m}) 6$ months after initiation of treatment $(p<0.001)$ and to $249.0 \mu \mathrm{m}$ (interquartile range, $213.8-311.0 \mu \mathrm{m}) 12$ months after initiation of treatment $(p$ $<0.001$ ) (Fig. 1A, 1B).

\section{Treatment exposure}

Following the initial IAI, CRT decreased below $300 \mu \mathrm{m}$ in 14 eyes (46.7\%); after up to five consecutive IAI administrations, CRT decreased below $300 \mu \mathrm{m}$ in 20 eyes (66.7\%) (Fig. 2). After six or more consecutive IAI administrations, CRT initially decreased below $300 \mu \mathrm{m}$ in six eyes $(20.0 \%)$ but never decreased below $300 \mu \mathrm{m}$ in four eyes (13.3\%) during the follow-up period. Table 3 lists the differences in baseline characteristics for subgroups of patients who responded differently to the IAIs in terms of first observed decrease of CRT below $300 \mu \mathrm{m}$ or the lack of such response; we found no significant differences in baseline characteristics among the listed subgroups. The median number of IAIs administered to a patient during the 12 months was 6.0 (interquartile range, 5.0-9.0).

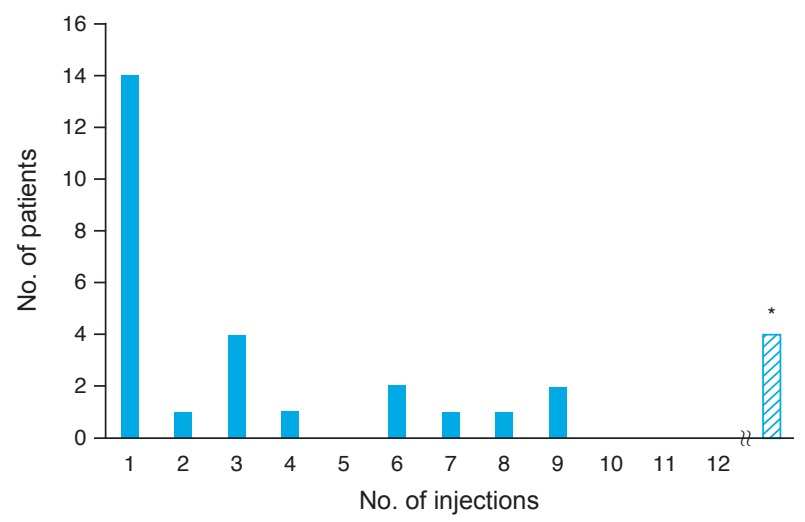

Fig. 2. Number of intravitreous aflibercept injections until central retinal thickness (CRT) initially reduced to $<300 \mu \mathrm{m}(\mathrm{n}=30)$. "The number of the patients in which CRT never reduced to $<300$ $\mu \mathrm{m}$, including the patients in which CRT never reduced to $<300$ $\mu \mathrm{m}$ at the time of dropout. 
Table 3. Baseline characteristics depending on the number of IAIs administered until CRT first decreased below $300 \mu \mathrm{m}$

\begin{tabular}{|c|c|c|c|c|}
\hline & $\begin{array}{l}\text { CRT decreased below } 300 \mu \mathrm{m} \\
\text { after up to } 5 \text { consecutive } \\
\text { IAIs }(\mathrm{n}=20)\end{array}$ & $\begin{array}{l}\text { CRT decreased below } 300 \mu \mathrm{m} \\
\text { after } 6 \text { or more consecutive } \\
\text { IAIs }(\mathrm{n}=6)\end{array}$ & $\begin{array}{l}\text { CRT never decreased below } \\
\qquad 300 \mu \mathrm{m}(\mathrm{n}=4)\end{array}$ & $p$-value \\
\hline Age (yr) & $70.0(64.5-74.5)$ & $64.5(60.0-71.3)$ & $69.0(65.0-74.0)$ & $0.721^{*}$ \\
\hline Sex, female & $7(35.0)$ & $1(16.7)$ & $2(50.0)$ & $0.525^{\dagger}$ \\
\hline Phakia & $13(65.0)$ & $6(100.0)$ & $3(75.0)$ & $0.267^{\dagger}$ \\
\hline HbA1c (\%) & $6.7(6.5-7.6)$ & $7.0(6.8-7.4)$ & $7.2(6.5-8.1)$ & $0.682^{*}$ \\
\hline $\mathrm{eGFR}\left(\mathrm{mL} / \mathrm{min} / 1.73 \mathrm{~m}^{2}\right)$ & $53.5(43.6-70.3)$ & $51.7(44.4-63.3)$ & $60.2(53.1-63.4)$ & $0.935^{*}$ \\
\hline History of PRP & $8(40.0)$ & $1(16.7)$ & $0(0.0)$ & $0.290^{\dagger}$ \\
\hline $\begin{array}{l}\text { DRSS score } \geq 53 \\
\text { at baseline }\end{array}$ & $13(65.0)$ & $3(50.0)$ & $1(25.0)$ & $0.303^{\dagger}$ \\
\hline BCVA (logMAR) & $0.61(0.40-0.73)$ & $0.35(0.30-0.49)$ & $0.40(0.35-0.43)$ & $0.120^{*}$ \\
\hline $\mathrm{CRT}(\mu \mathrm{m})$ & $410.0(364.0-492.3)$ & $484.5(446.3-556.5)$ & $417.0(384.0-449.3)$ & $0.242^{*}$ \\
\hline
\end{tabular}

Values are presented as median (interquartile range) or number (\%).

IAI = intravitreal aflibercept injection; CRT = central retinal thickness; HbAlc = glycated hemoglobin; eGFR = estimated glomerular filtration rate; $\mathrm{PRP}=$ panretinal photocoagulation; $\mathrm{DRSS}=$ diabetic retinopathy severity scale; BCVA = best-corrected visual acuity; log$\mathrm{MAR}=$ logarithm of the minimum angle of resolution.

${ }^{*}$ Kruskal-Wallis test; 'Fisher exact test.

Table 4. Effect on DRSS score of aflibercept treat-and-extend regimen for the 25 patients who completed the 12-month treatment course

\begin{tabular}{lcc}
\hline & Month 6 & Month 12 \\
\hline Diabetic retinopathy worsening & $0(0)$ & $0(0)$ \\
No change & $21(84)$ & $17(68)$ \\
1 step improvement & $3(12)$ & $4(16)$ \\
2 step improvement & $1(4)$ & $4(16)$ \\
$\geq 3$ step improvement & $0(0)$ & $0(0)$ \\
\hline
\end{tabular}

Values are presented as number of patients (\%).

DRSS $=$ diabetic retinopathy severity scale.

\section{DRSS score}

Table 4 summarizes the effects of the aflibercept TAE regimen on DRSS scores. With treatment, there were some cases showing improved DRSS score. Compared to baseline, the DRSS score at 12 months was unchanged for $68 \%$ of patients, improved by one step for $16 \%$ of patient, and by two steps for $16 \%$ of patients, and in no cases did the DRSS score worsen or improve by three or more steps.

\section{Discussion}

A recent randomized clinical trial involving 650 neovascular age-related macular degeneration patients revealed that the use of a TAE regimen to administer the anti-VEGF agent ranibizumab for improvement of VA was statistically equivalent to and clinically comparable with a monthly treatment regimen [17]. Thus, it is expected that the use of a specific TAE regimen for administration of anti-VEGF agents will become more widespread, for patients afflicted with DME.

There have been two previous prospective clinical trials of ranibizumab for DME via TAE regimen $[18,19]$. In the TREX-DME study [18], all enrolled eyes received monthly intravitreal injection of ranibizumab for at least 4 months. The TAE algorithm used in that study was somewhat more complicated than the present one and determined whether the administration interval was extended, maintained, or shortened based on the proportion of changes from baseline. In the RETAIN study [19], all enrolled eyes received at least 3 monthly injections or until BCVA stability was achieved; determination of BCVA stability was at the discretion of the assessing clinician, with no predetermined set of criteria. 
In our TAE regimen, all enrolled eyes received at least 2 monthly injections, and the administration interval was determined according to CRT greater or less than $300 \mu \mathrm{m}$ based on OCT examination. We consider this regimen to be versatile because CRT is an objective numerical value and because our regimen does not require a complicated algorithm or specialist judgment.

Currently, a loading phase of 5 monthly injections is recommended when using aflibercept for the treatment of DME $[6,20]$. In the present prospective study, our findings illustrate the efficacy of aflibercept for DME via a TAE regimen. After five or fewer consecutive IAI administrations, CRT decreased below $300 \mu \mathrm{m}$ in $66.7 \%$ of the patients. However, after six or more consecutive IAI administrations, CRT initially decreased below $300 \mu \mathrm{m}$ in a further $20.0 \%$ of the patients. Pieramici et al. [21] previously reported that some patients have a limited early response to IAIs and studied patients in three subgroups of limited early response: those who met anatomic criteria (i.e., a CRT reduction less than or equal to $10 \%$ and CRT greater than $300 \mu \mathrm{m}$ ); those who met the BCVA criterion (i.e., an increase of BCVA less than five letters); and those who met both the anatomic and BCVA criteria. Overall, significant vision improvements were observed through week 100 with continued IAI treatment for a small number of the DME eyes that showed limited early response after 3 monthly IAIs. The present results also showed cases of delayed and sufficient anatomic improvement with continued IAI. Our regimen may help to reduce the number of IAIs administered to patients that respond well to IAI and not overlook the cases that respond more slowly.

In this present study, compared to baseline, the DRSS score at 12 months was improved by two steps in $16 \%$ of the patients, and in no cases did the DRSS score worsen during the follow-up period. Mitchell et al. [22] previously reported that IAIs for DME improved the DRSS score of some patients. At the 2019 Annual Meeting of the Association for Research in Vision and Ophthalmology in Vancouver, Boyer showed that IAI may reverse disease progression in patients diagnosed with moderately severe to severe nonproliferative DR. The US Food and Drug Administration recently approved aflibercept for treatment of all stages of DR. Although IAI is not approved for DR in Japan, our findings suggest that IAI might be effective for DR in patients with DME.

The present study has some notable limitations. First, it was performed as an uncontrolled and noncomparative study. Therefore, it cannot be compared with other administration regimens such as monthly injections or pro re nata use. Second, the sample size in this study was relatively small. Therefore, it cannot illustrate the factors that influence the therapeutic effect of IAI. Because it is desirable to minimize the number of intravitreal injections of anti-VEGF agent, various combination therapies have been reported [18,23]. Hence, further investigations are needed to elucidate the ideal IAI administration method.

In conclusion, the findings of this study show that, when administered as a TAE regimen, aflibercept is an effective treatment option for DME.

\section{Conflict of Interest}

This investigator-initiated research study was supported by Bayer Yakuhin, Ltd. (Osaka, Japan). Bayer Yakuhin, Ltd. had no role in the study design or execution. No other potential conflicts of interest relevant to this article were reported.

\section{Acknowledgements}

The authors wish to thank John Bush for editing the manuscript.

\section{References}

1. Murata T, Ishibashi T, Khalil A, et al. Vascular endothelial growth factor plays a role in hyperpermeability of diabetic retinal vessels. Ophthalmic Res 1995;27:48-52.

2. Diabetic Retinopathy Clinical Research Network, Wells JA, Glassman AR, et al. Aflibercept, bevacizumab, or ranibizumab for diabetic macular edema. $N$ Engl J Med 2015;372:1193-203.

3. Lanzetta P, Loewenstein A; Vision Academy Steering Committee. Fundamental principles of an anti-VEGF treatment regimen: optimal application of intravitreal anti-vascular endothelial growth factor therapy of macular diseases. Graefes Arch Clin Exp Ophthalmol 2017;255:1259-73.

4. Sugimoto M, Tsukitome H, Okamoto F, et al. Clinical preferences and trends of anti-vascular endothelial growth fac- 
tor treatments for diabetic macular edema in Japan. $J$ Diabetes Investig 2019;10:475-83.

5. Nguyen QD, Brown DM, Marcus DM, et al. Ranibizumab for diabetic macular edema: results from 2 phase III randomized trials: RISE and RIDE. Ophthalmology 2012;119:789-801.

6. Korobelnik JF, Do DV, Schmidt-Erfurth U, et al. Intravitreal aflibercept for diabetic macular edema. Ophthalmology 2014;121:2247-54.

7. Kim LN, Mehta H, Barthelmes D, et al. Metaanalysis of real-world outcomes of intravitreal ranibizumab for the treatment of neovascular age-related macular degeneration. Retina 2016;36:1418-31.

8. Zhang Y, Chioreso C, Schweizer ML, Abramoff MD. Effects of aflibercept for neovascular age-related macular degeneration: a systematic review and meta-analysis of observational comparative studies. Invest Ophthalmol Vis Sci 2017;58:5616-27.

9. Ashraf M, Souka A, Adelman RA. Age-related macular degeneration: using morphological predictors to modify current treatment protocols. Acta Ophthalmol 2018;96:12033.

10. 1Freund KB, Korobelnik JF, Devenyi R, et al. Treat-andextend regimens with anti-vegf agents in retinal diseases: a literature review and consensus recommendations. Retina 2015;35:1489-506.

11. Chin-Yee D, Eck T, Fowler S, et al. A systematic review of as needed versus treat and extend ranibizumab or bevacizumab treatment regimens for neovascular age-related macular degeneration. Br J Ophthalmol 2016;100:914-7.

12. Stewart MW. Aflibercept (VEGF Trap-eye): the newest anti-VEGF drug. Br J Ophthalmol 2012;96:1157-8.

13. Kanda A, Noda K, Saito W, Ishida S. Aflibercept traps galectin-1, an angiogenic factor associated with diabetic retinopathy. Sci Rep 2015;5:17946.

14. Grading diabetic retinopathy from stereoscopic color fundus photographs: an extension of the modified Airlie House classification. ETDRS report number 10. Early Treatment
Diabetic Retinopathy Study Research Group. Ophthalmology 1991;98:786-806.

15. Ip MS, Domalpally A, Hopkins JJ, et al. Long-term effects of ranibizumab on diabetic retinopathy severity and progression. Arch Ophthalmol 2012;130:1145-52.

16. Staurenghi G, Feltgen N, Arnold JJ, et al. Impact of baseline diabetic retinopathy severity scale scores on visual outcomes in the VIVID-DME and VISTA-DME studies. Br J Ophthalmol 2018;102:954-8.

17. Silva R, Berta A, Larsen M, et al. Treat-and-extend versus monthly regimen in neovascular age-related macular degeneration: results with ranibizumab from the TREND Study. Ophthalmology 2018;125:57-65.

18. Payne JF, Wykoff CC, Clark WL, et al. Randomized trial of treat and extend ranibizumab with and without navigated laser for diabetic macular edema: TREX-DME 1 year outcomes. Ophthalmology 2017;124:74-81.

19. Prunte C, Fajnkuchen F, Mahmood S, et al. Ranibizumab $0.5 \mathrm{mg}$ treat-and-extend regimen for diabetic macular oedema: the RETAIN study. Br J Ophthalmol 2016;100:78795.

20. Avitabile T, Azzolini C, Bandello F, et al. Aflibercept in the treatment of diabetic macular edema: a review and consensus paper. Eur J Ophthalmol 2017;27:627-39.

21. Pieramici D, Singh RP, Gibson A, et al. Outcomes of diabetic macular edema eyes with limited early response in the VISTA and VIVID studies. Ophthalmol Retina 2018;2:55866.

22. Mitchell P, McAllister I, Larsen M, et al. Evaluating the impact of intravitreal aflibercept on diabetic retinopathy progression in the VIVID-DME and VISTA-DME studies. Ophthalmol Retina 2018;2:988-96.

23. Hirano T, Toriyama Y, Iesato Y, et al. Effect of leaking perifoveal microaneurysms on resolution of diabetic macular edema treated by combination therapy using anti-vascular endothelial growth factor and short pulse focal/grid laser photocoagulation. Jpn J Ophthalmol 2017;61:51-60. 